\title{
A catalog of the types of Geotrupidae, Glaresidae, Hybosoridae, Lucanidae and Trogidae (Insecta: Coleoptera: Scarabaeoidea) deposited in the Museo Argentino de Ciencias Naturales, Buenos Aires
}

\author{
Axel O. BACHMANN ${ }^{1}$, Rocío A. GONZALEZ-VAQUERO ${ }^{1} \&$ Federico C. OCAMPO ${ }^{2}$ \\ ${ }^{1}$ Entomology División, Museo Argentino de Ciencias Naturales «Bernardino Rivadavia». \\ bachmann 9 bg.fcen.uba.ar; rocioagv 9 yahoo.com. ${ }^{2}$ Entomology Laboratory, Instituto Argentino de \\ Investigaciones de Zonas Aridas, CC 507, 5500, Mendoza, Argentina. federico.ocampo $\odot$ gmail.com
}

\begin{abstract}
A catalog of the types, all current categories, of Geotrupidae, Glaresidae, Hybosoridae, Lucanidae and Trogidae, housed in this Museum, is given; it comprises the types of 40 names; 39 (98\%) of them are here represented by name-bearing types («primary types»).
\end{abstract}

Key words: scarab beetles, type specimens, museum collection, Argentina.

Resumen: Catálogo de los tipos de Geotrupidae, Glaresidae, Hybosoridae, Lucanidae y Trogidae (Insecta: Coleoptera: Scarabaeoidea) depositados en el Museo Argentino de Ciencias Naturales, Buenos Aires. Se catalogan los ejemplares típicos de todas las categorías aceptadas, de Geotrupidae, Glaresidae, Hybosoridae, Lucanidae y Trogidae, depositados en este museo; se registran 40 nombres; 39 de ellos (98\%) representados aquí por tipos portadores de nombres («tipos primarios»).

Palabras clave: escarabeidos, especímenes tipo, colección de museo, Argentina.

\section{INTRODUCTION}

A catalog of the type specimens of the Scarabaeoid families except Scarabaeidae s. l. housed in the Entomological Division of the Museo Argentino de Ciencias Naturales (MACN) is given. They correspond to: 21 Geotrupidae, two Glaresidae, 14 Hybosoridae, one Lucanidae, and two Trogidae; no types of Ochodaeidae nor Passalidae are currently housed at MACN. They correspond to 40 names: 31 holotypes, 20 syntypes (of six names), and 47 paratypes, these including 18 «allotypes». It follows that 39 names $(98 \%)$ are represented by name-bearing types («primary types»).

Specific and subspecific names are alphabetically entered, as it is usual in type specimen catalogs; each name is followed by the generic name (and subgeneric one if it was mentioned) as they were spelled in the original binomen, or by the generic and specific ones in the case of subspecies. The bibliographic citation follows, as well as an account of the types, if these were mentioned. If the country is not mentioned in the text, Argentina is meant. Nomenclatural or taxonomical changes that followed the original description are added, as they are known to the authors of this contribution. If no types were formally designated, and the amount of specimens examinated was not originally indicated, it is assumed that they are a syntype series (recommendation $73 \mathrm{~F}$ of the Code), eventually «single syntypes». For this contribution the classification proposed by Lawrence \& Newton (1995) is followed.

In the collection some specimens were not found; however, in order to assist in future research, they are mentioned in a separate list. Some specimens were labelled as types of names not found in the literature and assumed not to have been published. These names are probably not available in the sense of the ICZN (1999); however, also in order to assist in future research, they are mentioned in a third list. Specimens designated as types (mainly «allotypes») after the publication date, published or not, cannot be accepted as included in the type series; they are included in a further list, along with specimens labelled as types and not mentioned in the original publication. In every case, a Note is added to make clear of their condition.

Some specimens bear a registration number, which corresponds to the old registry books of the Entomological Division of the Museum. All specimens bear a new accession number with the acronym of the collection (e.g., «MACN-En 800»), which corresponds to the data-base of the $\mathrm{Mu}$ seum. The specimens that were not found, were 
not entered in the data-base. Specimen information is currently managed with the application Aurora (Rodríguez, 2007-2010).

Museum and collections are as follows: MACN, Museo Argentino de Ciencias Naturales Bernardino Rivadavia, Buenos Aires, Argentina; AMNH, American Museum of Natural History, New York, USA; BMNH, Berlin Museum of Natural History, Berlin, Germany; BR, Bruch coll., in part in MACN; CC, Carcavallo coll.; CR, Richter coll., in MLP; CSC, Campos Seabra coll.; CWC, Cartwright coll.; DC, Diringshofen coll.; FC, Fritz coll.; GC, Gutiérrez coll.; HB, Burmeister coll., in MACN; HC, Howden coll.; IML, Fundación e Instituto Miguel Lillo, Tucumán, Argentina; ISM, Instituto Entomológico de San Miguel, later INESALT, now in Salta National University, Salta, Argentina; MC, Martínez coll., types in MACN, remaining in HC; MEC, Melzer coll.; MF, Museum Frey, Tützing, Germany, now in NHMB; MLP, Museo de La Plata, La Plata, Argentina; MP, Museu Paulista, Universidade de São Paulo, São Paulo, Brasil; MZSP, Museu de Zoologia, São Paulo, Brasil; NHMB, Natural History Museum of Basel, Basel, Switzerland; PEC, Pereira coll.; PRC, Prosen coll.; RC, Robinson coll.; SC, Schajovskoy coll.; USNM, United States National Museum (Smithsonian Institution), Washington, USA.

Other abbreviations used are: allot., allotype; coll., collection; handwr., handwritten; holot., holotype; parat., paratype; prov., province; spec., specimen; synt., syntype; AOB, Axel O. Bachmann; FCO, Federico C. Ocampo.

\section{CATALOG OF THE TYPES}

\section{Geotrupidae}

acuticornis [Athyreus]. Howden \& Martínez 1978: 44-45, f. 52-54: Brasil: Villa Braga, leg. Howden, Holot. $0^{x}$; Pernambuco: Taperá, leg. Martínez, allot. $\odot$, leg. Martínez; paratypes, R. G. Norte: Natal, Pará: Belém, Paraíba, leg. Howden \& Martínez.

1 parat. \& , pinned, «Natal / R. G. Norte Brasil (printed) / 5-1-1951 / Sgt. Rodney (handwr.)» on white paper, with a black frame; «Coleção / M. Alvarenga» printed; «Allotypus» printed on pale red paper; «Allotype (printed) / Athyreus / acuticornis / Howden + Martínez (handwr.)» on red paper. MACN-En 819.

Note: The specimen is labelled "Allotype», but in the original publication it is mentioned as a paratype.

alvarengai [Athyreus]. Howden \& Martínez 1978: 31-32, f. 34-36, map 4: Brasil: Pernambuco:
Escada, Holot. ○’, leg. Martínez; paratypes, Pará, leg. Howden.

Holot. $\sigma^{x}$, glued to a card, genitalia and abdomen glued to another card, "Escada / Pernambuco / Brasil / X / A. Maller» handwr. on white paper, with a black frame; «Holotypus» printed on pale red paper; «Holotype (printed) / Athyreus / alvarengai / Howden + Martínez (handwr.)» on red paper; «SC / Per- / nam / buco / Escada / X» handwr. in pencil, folded in three. MACN-En 800.

Note: The collector on the label differs from the original publication.

arribalzagai [Athyreus]. Martínez 1951: 109112, f. 1a-c. Holot. from Corrientes, allot. from Paraguay, 1 parat. $\bigcirc$ from Paraguay: Villarrica, IV-1926, leg. Jörgensen, 52630, in MACN; other paratypes from Paraguay and Corrientes in MC; another paratype in ISM.

Holot. $\sigma^{7}$, pinned, genitalia glued to a card; «Argentina / Corrientes / Do․ Ituzaingó / I. Apipé Grande / Coll. Martínez / Nov. 945» handwr. on white paper; a rectangle of green paper; «Holotypus» printed on pale red paper, with a black frame; "Athyreus / arribalzagai o" / sp. n. (handwr.) / A. Martínez det. 1951 (printed)» on red paper, with a black frame. Allot. $\$$, glued to a card, abdomen glued to another card; "Paraguay / Do Villarrica / Cnel. Martínez / F. Schadeleg. / Coll. Martínez / Dic. 943» handwr. on white paper; «Allotypus» printed on pale red paper, with a black frame; «Athyreus / arribalzagai $\odot$ / sp. n. (handwr.) / A. Martínez det. 1951 (printed)» on red paper, with a black frame. 1 parat. $ᄋ$, glued to a card, abdomen glued to another card; «Paraguay / Villarrica / IX-23» handwr. on white paper; «52630" handwr. on white paper; "Paratipo $ᄋ$ » handwr. on green paper; "Athyreus / arribalzagai o / sp. n. (handwr.) / A. Martínez det. 1951 (printed)» on green paper, with a black frame. MACN-En 847, 848, 846 respectively.

Note: Currently in Neoathyreus (Howden \& Martínez 1978: 53).

bilobus [Athyreus]. Howden \& Martínez 1978: 26-27, f. 16-18, map 2: Brasil: Paraná: Arapongas, holot. $\sigma^{x}$, allot. $ᄋ$ in MC; paratypes from Brasil: Paraná, São Paulo, R. Janeiro, Minas Gerais and Goíás States, and from Paraguay, in several institutions.

Holot. $\sigma^{7}$, glued to a card, genitalia glued to another card; «Arapongas / Paraná Brasil / XII1951 / Anton Maller» printed on white paper, date handwr., with a black frame; "Arapongas / Paraná / 11.51» handwr. in pencil, folded in eight; «Holotype (printed) / Athyreus / bilobus / Howden 
+ Martínez (handwr.)» on red paper. Allot. \& , glued to a card; «Arapongas / Paraná Brasil / XII1951 / Anton Maller» printed on white paper, date handwr., with a black frame; «SC / Athyreus / 12.51» handwr. in pencil, folded in eight; "Allotype (printed) / Athyreus / bilobus / Howden + Martínez (handwr.)» on red paper. MACN-En 894, 895 respectively.

borgmeieri [Bolbapium]. Martínez 1976: 539542, 546, f. 5-6: Brasil: Goiás: Rio Verde, holot. $\sigma^{7}$, allot. $ᄋ$, 1 parat. $\sigma^{\pi}$ all in MC.

Holot. $\sigma^{"}$, glued to a card; «Brasil / Eo Goiaz / Rio Verde / Zellibar leg. / Coll. Martínez / Dic. 945» handwr. on white paper; «Brasil / Goiaz Rio Verde / Zellibar / sp. nov.» handwr. on translucent paper, folded in eight; "Holotypus» printed on pale red

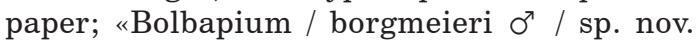
(handwr.) / A. Martínez det. 1956 (printed)» on red paper, with a black frame. MACN-En 924.

cantisanii [Bolborhinum (Zefevazia)]. Martínez 1976: 535-538, f. 3-4: Buenos Aires: Puán: Felipe Solá, holot. $\sigma^{7}$, allot. $\odot$ both in MC; paratypes from Buenos Aires, Río Negro, Mendoza, La Rioja, and Córdoba provinces in BR, IML and MF.

Holot. $\sigma^{x}$, pinned, genitalia and abdomen glued to two cards; «Argentina / Buenos Aires / Pdo. Puan / Felipe Solá / Coll. Martínez / Ene. 952» handwr. on white paper; «Zefevazia / peruana $0^{\pi} /$ (Boucom.) (handwr.) / A. Martínez det. 1954 (printed)» on white paper, with a black frame; "Holotypus» printed on pink paper; «Bolborh. (Zef.) / cantisanii o / sp. nov. (handwr.) / A. Martínez det. 1976 (printed)» on red paper, with a black frame. Allot. $\%$, pinned, abdomen glued to a card; «Argentina / Buenos Aires / Pdo. Puan / Est. Felipe Solá $\odot$ / Coll. Martínez» handwr. on white paper; «Allotypus» printed on pale red paper; "Bolborhinum (Zefevazia) / cantisanii $\circ$ / sp. nov. (handwr.) / A. Martínez det. 1976 (printed)» on red paper, with a black frame. MACN-En 972, 971 respectively.

Note: The specimen was misidentified as Zefevazia peruana (Boucomont).

capricornis [Athyreus]. Howden \& Martínez 1978: 21-22: Brasil: São Paulo: Batatais, holot. $\sigma^{7}$, allot. $\odot$, both in MC.

Holot. $\sigma^{7}$, pinned, genitalia glued to a card; «Batatais E. de S. Paulo / Brasil / V.945 / Col. do Ginasio S. José» printed on white paper, date handwr.; «Athyreus / cyanescens / Klug (handwr.) / P. Pereira det. 945 (printed)» on white paper, with a black frame; "Holotype (printed) / Athyreus / capricornis / Howden + Martínez (handwr.)» on red paper. MACN-En 975. conspicuus [Athyreus]. Howden \& Martínez 1978: 51-52, f. 63, map 9: Santiago del Estero: Río Salado: Icaño, holot. ㅇ in MC; paratypes from Santiago del Estero and La Rioja Provinces in MACN-En, IML, leg. Martínez and leg. Howden.

Holot. 옹 glued to a card, abdomen glued to another card; «Sgo. del Estero / Río Salado / Wagner col.» printed on white paper, with a black frame; "Holotype (printed) / Athyreus / conspicuus / Howden + Martínez (handwr.)» on red paper. MACN-En 1014.

howdeni [Bolbapium]. Martínez 1976: 542-454, 546, f. 7-8: Bolivia: S. Cruz: Ichilo: Buenavista, y prov. Sara: S. Rosa, holot. $\sigma^{7}, 1$ parat. $\sigma^{7}$ all in MC.

Holot. $\sigma^{x}$, glued to a card, genitalia and abdomen glued to another card; «Bolivia / Dep. Sta. Cruz / Prov. Ichilo / Buenavista / Coll. Martínez / Oct. 949» handwr. on white paper; «Holotypus» printed on pale red paper; «Bolbapium / howdeni $\sigma^{7} /$ sp. nov. (handwr.) / A. Martínez det. 1976 (printed)» on red paper, with a black frame. MACN-En 1136.

hypocritus [Athyreus]. Howden \& Martínez 1978: 24-25, f. 13-15, map 2: Formosa: Clorinda, holot. $\sigma^{x}$; Paraguay: Santa Bárbara, allot. $ᄋ$, both in MC; paratypes from Argentina and Paraguay, in $\mathrm{MC}$ and $\mathrm{HC}$.

Holot. $\sigma^{7}$, glued to a card, genitalia glued to another card; «Argentina / Formosa / Clorinda / Coll. Martínez / Nov. 949» handwr. on white paper; «Holotype (printed) / Athyreus / hypocritus / Howden + Martínez (handwr.)» on red paper. Allot. \& , glued to a card; «Paraguay / Sta. Bárbara / Schade-legit. / Coll. Martínez / 2.III.948» handwr. on white paper; «Allotype (printed) / Athyreus / hypocritus / Howden + / Martínez (handwr.)» on red paper. MACN-En 1142,1143 respectively.

juanae [Athyreus]. Howden \& Martínez 1978: 41, f. 61-62, map 6: Brasil: Guaporé: Porto Velho, holot. $\sigma^{7}$ in MC, parat. in HC.

Holot. $\sigma^{7}$, pinned, genitalia and abdomen glued to a card; «Porto Velho / Territorio Guaporé Brasil / II-1945 / A. Parko» printed on white paper, date handwr.; «M.P.P.Velho / S. Carlos / Guaporé / II-1945 / A. Parko» handwr. in pencil; «Coleção Campos Seabra» printed; «Holotype (printed) / Athyreus / juanae / Howden + Martínez (handwr.)» on red paper. MACN-En 1170 .

lanei [Athyreus]. Martínez 1952: 110-118, f. 39ab, 40: Venezuela: Distrito Federal: Cerro del Naiguatá, 720 m, VII-1943, VII-1945 leg. Lichy; 


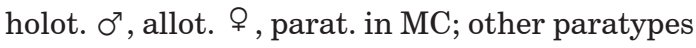
in MP, PRC, GC and RC.

Holot. $\sigma^{x}$, glued to a card, genitalia and abdomen glued to another card; «Venezuela / D. F. 720 mts. Alt. / Co Naiguatá / R. Lichy-legit / Coll. Martínez / Jul. 945» handwr. on white paper; «Holotypus» printed on pale red paper; «Athyreus / lanei ơ / sp. n. (handwr.) / A. Martínez det. 1952 (printed) « on red paper, with a black frame. Al-


«Venezuela / D. F. 720 mts. Alt. / Co Naiguatá / R. Lichy-legit / Coll. Martínez / Julio 945» handwr. on white paper; «Allotypus» printed on pale red paper; «Athyreus / lanei ㅇ / sp. n. (handwr.) / A. Martínez det. 1952 (printed) " on red paper, with a black frame. 1 parat. $\$$, glued to a card, abdomen on another card; «Venezuela / D. F. $720 \mathrm{mts}$. Alt. / Co Naiguatá / R. Lichy-legit / Coll. Martínez / Julio 945» handwr. on white paper; «Paratipo ㅇ » handwr. on green paper; «Athyreus / lanei 우 / sp. n. (handwr.) / A. Martínez det. 1952 (printed) " on green paper, with a black frame. MACN-En $1229,1230,1228$ respectively.

Note: Currently in Neoathyreus (Howden \& Martínez 1978: 53).

minutum [Bolboceras]. Luederwaldt 1929: 71: Brasil: Matto Grosso, 1 specimen in MEC.

Holot., glued to a card, abdomen on the same card; «Matto Grosso / Murtinho / XI.1927 / W. Melzer» handwr. on white paper; "Bolboceras / minutum / Lueder / Lueder det. 29» handwr. on white paper, with a black frame; «Bolboceras / minutum / Luderw.» handwr. on white paper; «Bolboceras / minuta / Luedw.» handwr. on white paper; «Holotypus» printed on pale red paper; a dark blue disc pinned. MACN-En 1296.

Note: Currently in Bolbapium Boucomont (Martínez 1976: 546).

nitidus [Athyreus]. Howden \& Martínez 1978: 29-30, f. 21-22, map 3: Bolivia: Santa Cruz: Río Seco, holot. $\sigma^{7}$ in MC.

Holot. $\sigma^{7}$, glued to a card, genitalia glued to another card; «Bolivia / Do Sta. Cruz / Pcia. Cordillera / Río Seco / Coll. Martinez / Feb. 962» handwr. on white paper; "nitidus» handwr. in pencil; «Holotype (printed) / Athyreus / juanae / Howden + Martínez (handwr.)» on red paper. MACN-En 1324.

quadrispinosum [Bolboceras]. Luederwaldt 1929: 71-72: Brasil: São Paulo, 2 specimens in MEC.

1 synt., glued to a card; «Typus» printed on pale red paper; «Typo!» handwr. on white paper; «Taquaritinga / Dez. 1914 / (something ilegible)
St. Ans» handwr. on white paper; «Bolboceras / 4-spinosum / Lüderw.» handwr.; «Bolboceras / 4spinosum / Lueder / Luederw. Det. 29» handwr. on white paper, with a black frame; «Bolboceras / quadrispi- / nosa Luedw.» handwr. MACN-En 1431.

Note: Currently in Bolbapium Boucomont (Martínez 1976: 547).

riojanum [Bolboceras]. Martínez 1951: 117-120, f. 3a,b: Holot. and 3 parat. $\sigma^{7}$ from La Rioja: La Cañada, III-1946, in MC; 2 parat. $\sigma^{7}$ from La Rioja: Patquía, 52631, in MACN.

Holot. $\sigma^{\prime}$, pinned; «Argentina / La Rioja / D ${ }^{\circ}$ Capital / La Cañada / Coll. Martínez / Marz. 946» handwr. on white paper; «Holotypus» printed on pale red paper, with a black frame; «Bolboceras / riojanum $0^{7}$ / sp. n. (handwr.) / A. Martínez det. 1951 (printed) « on red paper, with a black frame. 2 parat. $\sigma^{\prime}$, pinned, genitalia glued to a card; «La Rioja / Patquía / leg. Breyer» handwr. on white paper; «Col. A. Breyer» printed; «52631»; «Paratipo o"» handwr. on green paper; «Bolboceras / riojanum / sp. n. ơ (handwr.) / A. Martínez det. 1951 (printed) " on green paper, with a black frame. MACN-En 1444, 1442, 1443 respectively.

Note: Currently in Halffterobolbus (Martínez 1976: 548).

rosascostai [Zefevazia]. Martínez 1954: 198, 200-204, f. 1-9: Santiago del Estero: Termas de Río Hondo; Córdoba: Villa Dolores, holot. $\sigma^{7}$, allot. ㅇ , parat. 6 ㅇ , $60^{7}$ in $\mathrm{MC}, 10^{7}$ in PEC, $10^{7}$ in $\mathrm{HC}, 1$ i in CWC.

Holot. $\sigma^{x}$, allot $\odot$, glued to cards; "Argentina / Sgo. del Estero / Río Hondo / A. Ogloblinleg. / Coll. Martínez / 25-XI-53» handwr. on white paper; the holot: «Holotypus» printed on pale red paper, with a black frame; "Zefevazia / rosascostai $\sigma^{7} /$ gen. et sp. n. (handwr.) / A. Martínez det. 1954 (printed)» on red paper, with a black frame; the allot: «Allotypus» printed on pale red paper, with a black frame; «Zefevazia / rosascostai ㅇ / gen. et sp. n. (handwr.) / A. Martínez det. 1954 (printed)» on red paper, with a black frame. MACN-En 1450, 1451 respectively.

Note: Currently in Bolborhinum (Zefevazia) (Martínez 1976: 549).

ruficollis [Athyreus]. Bruch 1925: 199-200, f. 1: Santiago del Estero, Wagner.

Holot., pinned, «Stgo. Estero / Río Salado / E. Wagner» handwr. by Bruch; «Typus» printed on pale green paper; "Athyreus / ruficollis / Bruch" handwr. by Bruch, with a red frame; «Kolbeus / ruficollis / m. (handwr. by Bruch) / C. Bruch determ. (printed)»; «Stenasbidius / 
ruficollis / (Bruch) (handwr.) / A. Martínez det. 1952 (printed)». MACN-En 1452.

Note: Currently in Bolbothyreus (Howden 1973: 1567).

seai [Bolboceras]. Martínez 1951: 113-116, f. 2ab: Catamarca: Ambato: Los Angeles, holot. $\sigma^{7}$, in MC.

Holot. $\sigma^{7}$, pinned, an antenna and tarsi glued on locality card; "Argentina / Catamarca / $\mathrm{D}^{\circ}$ Ambato / Los Angeles / B. Schafer-leg. / Feb. 946» handwr. on white paper; «Holotypus» printed on pale red paper, with a black frame; «Bolboceras / seai $\sigma^{7}$ / sp. n. (handwr.) / A. Martínez det. 1951 (printed)» on red paper, with a black frame. MACN-En 1464.

Note: Currently in Bolborhinum (Bolborhinum) (Martínez 1976: 548).

shajovskoyi [Bolboceras (Bolborhinum)]. Martínez 1952: 318-322, 326, f. 1-3: Neuquén: San Martín de los Andes, I-1950, I-1951, leg. Schajovskoy, holot. $0^{7}$, allot. $\odot$, parat. $60^{7}, 5$ ㅇ in MC; parat. in SC and PRC, 1 parat. $ᄋ$, 53268, in MACN.

Holot. $\sigma^{x}$, pinned, genitalia glued to a card; «S. M. Andes / P. Nac. Lanín / 1. Swaryezewski» photographed from typed on white paper; «Holotypus» printed on pale red paper, with a black frame; «Bolboceras (B.) / shajovskoyi $0^{\top} /$ sp. n. (handwr.) / A. Martínez det. 1952 (printed)» on red paper, with a black frame. Allot. $ᄋ$, pinned; "Enero 1950 / S. M. Andes / 1. Schajovskoi» photographed from typed on white paper; «Allotypus» printed on pale red paper, with a black frame; «Bolboceras (B.) / shajovskoyi ㅇ / sp. n. (handwr.) / A. Martínez det. 1952 (printed)» on red paper, with a black frame. 1 parat., pinned, «Rep. Argentina / Gob. Neuquén / C. Bruch» printed on white paper; «Col. C. Bruch» printed on white paper; «53268» handwr.; «Paratipo» printed on green paper; «Bolboceras (Bolborh.) / shajovskoyi + / sp. n. (handwr.) / A. Martínez det. 1952 (printed)» on green paper, with a black frame; in BR 66. MACN-En 1473, 1472, 1471 respectively.

Note: Currently in Bolborhinum (Bolborhinum) (Martínez 1976: 549).

zischkai [Athyreus]. Martínez 1953: 227-231, 232, pl 1: 1-2: Bolivia: Cochabamba: Prov. Chapare: Villa General Román, 400 m, holot. O", allot. \% , in MC.

Holot. $\sigma^{7}$, allot. $ᄋ$, pinned; holot.: «Bolivia / Chapare / $400 \mathrm{mts}$ altura / R. Zischka-leg. / Coll. Martínez» handwr. on white paper; «Holotypus» printed on pale red paper, with a black frame;
«Athyreus / zischkai o" / sp. n. (handwr.) / A. Martínez det. 1952 (printed)» on red paper, with a black frame; allot.: «Bolivia / Chapare / $400 \mathrm{M}$ / Zischka» printed on white paper, with a grey frame; "Bolivia / Región subandina / Prov. Chapare - $400 \mathrm{M}$ / ex Coll. Zischka» printed on white paper, with a black frame, folded, on the other side: " $\mathrm{N}^{\circ} 121 / 2^{\circ}$ envío» handwr.; «Allotypus» printed on pale red paper, with a black frame; "Athyreus / zischkai $ᄋ$ / sp. n. (handwr.) / A. Martínez det. 1952 (printed)» on red paper, with a black frame. MACN-En 1566, 1567 respectively.

\section{Glaresidae}

fritzi [Glaresis (Eoglaresis)]. Martínez, Pereira \& Vulcano 1961: 77-82: 3 ㅇ parat. Catamarca;

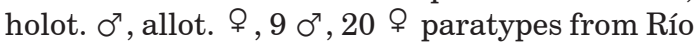
Negro: Lamarque, XI-1957, XI-XII-1958, leg. Fritz in MC and FC.

Holot. $\sigma^{x}$ and allot. $\circ$, glued to cards; «Argentina / Río Negro / Lamarque / Fritz - leg. / Coll. Martínez / Dic. 959» handwr. on white paper. The holot.: «Holotypus» printed on pale red paper; «Glaresis (Eogl.) / fritzi / sp. n. o" (handwr.) / A. Martínez det. 1961 (printed)» on red paper, with a black frame. The allot.: «Allotypus» printed on pale red paper; «Glaresis (Eogl.) / fritzi / sp. n. ㅇ (handwr.) / A. Martínez det. 1961 (printed)» on red paper, with a black frame. 3 parat., glued to cards; in two: «Fte. Quemado / Catam. Wolters» handwr. by Bruch on white paper; in the other one: «La Ciénaga (Belén) / Catamarca 11.1927 / Weiser Wolters leg.» Printed on white paper, date handwr. In the three parat. «Paratipo» printed on green paper, with a black frame; «Glaresis (Eogl.) / fritzi/sp. n. ㅇ (handwr.) / A. Martínez det. 1961 (printed)» on green paper, with a black frame. All parat. in BR 67 . MACN-En 1071, 1072, 1068-1070 respectively.

pardoalcaidei [Glaresis]. Martínez, Pereira \& Vulcano 1961: 69-77, f. 1-13, pl. 3: Holot., allot. and paratypes from Tucumán in MC, paratypes from Tucumán, Catamarca, Santiago del Estero, Chaco and Córdoba Provinces in BR. Other paratypes in MC, PRC, CC, PEC, CSC and DC.


talia $\sigma^{\pi}$ glued to another card; "Argentina / Tucumán / Do Capital [Ciudad] / Pte. Río Salí / Coll. Martínez / Nov. 950» handwr. on white paper; «Holotypus» and «Allotypus» printed on pale red paper; "Glaresis / pardo-alcaidei o" [ㅇ / sp. n. (handwr.) / A. Martínez det. 1961 (printed)» on red paper, with a black frame. 2 parat. + , glued to cards; «Rep. Argentina / Gob. Chaco / 
190_/ C. Bruch» printed on white paper; «Chärata / Bosq X.1924» handwr. by Bruch. 5 parat.: 3 우 in the same pin, 1 오, $1 \sigma^{\prime}$, all glued to cards; "Vilelas / Konstantinoff» handwr. by Bruch. 1 parat. ㅇ, glued to a card; «Rep. Argentina / Prov. Córdoba / $190 \_$/ C. Bruch» printed on white paper; «Weiser / legit.» handwr. by Bruch. 1 parat. $\%$, glued to a card; «Stgo. Estero» handwr. by Bruch. 6 parat., 5 ㅇ , $10^{7}$, glued to cards; «Rep. Argentina / Pr. Santiago d. Estero / 190-_/ C. Bruch» printed on white paper. 1 parat. $\sigma^{\star}$, pinned; «C. S. Reed / Catamarca» printed on white paper. 1 parat. $\sigma$, glued to a card; «Catamarca / 1917.» handwr. by Bruch. 1 parat. ๙", glued to a card; "Iliar Rioja / M. Gómez» handwr. by Bruch. 1 parat. $\sigma^{\top}$, glued to a card; «Rep. Argentina / Prov. Tucumán / 190__ / C. Bruch» printed on white paper. All parat. with «Paratipo» printed on green paper, with a black frame; "Glaresis / pardo-alcaidei / sp. n. ( $\sigma^{\star}$ or 우 accordingly) (handwr.) / A. Martínez det. 1961 (printed)» on green paper, with a black frame. All parat. in BR 67. MACN-En 1361, 1362, 1347 1360, 1111, 1164, 1288, 1289, 1582 respectively.

\section{Hybosoridae}

allsoppi [Chaetodus (Pseudohybosorus)]. Martínez 1988: 64-66, f. 1-3: Perú: Cusco: Santa Isabel, Valle Cosnipata, holot. $\sigma^{\top}$, allot. 우, in MC.

Holot. $\sigma^{\pi}$, glued to a card, genitalia glued to the same card; «Dept. Cusco, Perú / Santa Isabel / Cosnipata Valley/ Nov. 301951 / Felix Woytkowski» printed on white paper, date handwr.; «Holotipo» handwr. on red paper; «Chaetodus (Chaetodus) / allsoppi $\sigma^{\top}$ / sp. n. (handwr.) / A. Martínez det. 1987 (printed)» on red paper, with a black frame; «Bs As. / FCO 04/99» printed on yellow paper. MACNEn 797.

asuai [Chaetodus]. Martínez 1956: 43-44: Bolivia: Cochabamba: prov. Chapare, S. Francisco de Chipiriri, holot. $\sigma^{\pi}, 1 \sigma^{\pi}$ parat. in MC.

Holot. $\sigma^{x}$, glued to a card, genitalia glued to another card; «Bolivia Do Cochab. / Pcia. Chapare - S. F. del / Chipiriri 400 m. IV-53 / Martínez-col.» printed on white paper; «Holotypus» printed on pale red paper; "Chaetodus / asuai $\sigma^{\top} / \mathrm{sp}$. n. (handwr.) / A. Martínez det. 1956 (printed)» on red paper, with a black frame; «Bs As. / FCO 04/ 99» printed on yellow paper. MACN-En 851.

bolivianus [Chaetodus]. Martínez 1956: 41-43: Bolivia: Cochabamba: Prov. Chapare: Río Chapare, holot. $\sigma^{\top}$, allot. ㅇ , $4 \sigma^{\top}, 1$ 우 parat in MC.

Holot. $\sigma^{x}$, glued to a card, genitalia glued to another card, allot. 우, glued to a card; «Bolivia
/ Do Cochabamba / Prov. Chapare / Gral. Román, 450 mts / Coll. Martínez / Feb.952» handwr. on white paper; in the holot.: «Holotypus» printed on pale red paper; "Chaetodus / bolivianus $\sigma^{\top /}$ sp. n. (handwr.) / A. Martínez det. 1956 (printed)» on red paper, with a black frame; in the allot.: "Allotypus» printed on pale red paper; «Chaetodus / bolivianus 우 / sp. n. (handwr.) / A. Martínez det. 1956 (printed)» on red paper, with a black frame. MACN-En 910, 911 respectively.

brancucii [Chaetodus (Chaetodopsis)]. Martínez 1994: 224-227, f. 1: Venezuela: Terr. Fed. Amazonas, Dep. Atures: El Infierno y Coromoto, holot. $\sigma^{7}$, allot. 우, $5 \sigma^{7}, 7$ 우 parat. in MC.

Holot. $\sigma^{\gamma}$, glued to a card, genitalia glued to the same card; «Venezuela / T.F. Amazonas / Do Atures / Km 25 a Gavilán / El Infierno / Coll. Martínez / Jul. 979» handwr. on white paper; «Holotypus» printed on pale red paper; «Chaetodus / (Chaetodopsis) / brancucii o" / sp. nov. (handwr.) / A. Martínez det. 1987 (printed)» on red paper, with a black frame. Allot. 우, glued to a card, abdomen on the same card; «Venezuela / T.F. Amazonas / Do Atures / El Infierno / Km 25 a Gavilán / Coll. Martínez / Jul. 979» handwr. on white paper; «Allotypus» printed on pale red paper; «Chaetodus / (Chaetodopsis) / brancucii 우 / sp. nov. (handwr.) / A. Martínez det. 1987 (printed)» on red paper, with a black frame. MACN-En 929, 930 respectively.

cicheroi [Astaenomoechus]. Martínez 1970: 112114: Bolivia: Santa Cruz: prov. Sara: Nueva Moka, in Nasutitermes sp. nest, holot. 우 in MC.

Holot. $\odot$, glued to a card, abdomen glued to the same card; «Bolivia / Do Sta. Cruz / Pcia. Sara / Nueva Moka / Coll. Martínez / Jun. 1960» handwr. on white paper; «Holotypus» printed on pale red paper; «Astaenomoechus / cicheroi 우 / sp. nov. (handwr.) / A. Martínez det. 1969 (printed)» on red paper, with a black frame. MACN-En 1008.

fraternus [Chaetodus (Chaetodus)]. Martínez 1994: 230-232, f. 3-4: Bolivia: Santa Cruz: Ichilo: Buenavista, holot. $\sigma^{\top}$, allot. 우, $5 \sigma^{\top}, 7$ 우 paratypes in $\mathrm{MC}$.

Holot. $\sigma^{\pi}$, glued to a card, genitalia and abdomen glued to another card, allot. $ᄋ$, glued to a card, abdomen on the same card, next to the specimen. «Bolivia, Do Sta. / Cruz, Pcia. Ichilo, / Buenavista III-51- / Martínez leg.» photographed from typed on white paper; holot.: «Holotipo» handwr. on pink paper; «Chaetodus / (Barrochroides) / fraternus $\sigma^{7} / \mathrm{sp}$. nov. (handwr.) / A. Martínez det. 1987 (printed)» on red paper, 
with a black frame; allot.: «Allotypus» printed on pale red paper; «Chaetodus / (Barrochroides) / fraternus $\%$ / sp. nov. (handwr.) / A. Martínez det. 1987 (printed)» on red paper, with a black frame. MACN-En 1066, 1067 respectively.

Note: The subgenus on the labels differs from the original publication.

fuscoviridis [Acanthocerus]. Ohaus 1911: 553554: Misiones: Bonpland, Jörgensen.

2 synt., pinned; «Bonpland / Jörgensen» printed; «Typus» printed on pale green paper; in one «Acanthocerus / fuscoviridis / Ohaus» handwr. by Bruch, with a red frame; «Acanthocerus / fuscoviridis Ohaus / Type» handwr. by Ohaus, with «Ohaus determin. 1911» printed; in the other one «Acanthocerus / fuscoviridis / Ohaus 1911 / Syntypus» handwr. by AOB. Both in BR 64 . MACN-En 1074, 1075 respectively.

Note: Currently in Ceratocanthus (Martínez 1967: 12).

hamiger [Cloeotus]. Ohaus 1911: 554-555, f. 14: Syntypes $\sigma^{7}$ and $\odot$ from Córdoba and Santiago del Estero, leg. Bruch, in BR; from Chaco de Santiago del Estero: Río Salado, leg. Wagner, in BMNH.

1 synt. ㅇ , pinned; «Rep. Argentina / Prov. Córdoba / 16-II-1901 / C. Bruch» printed, date handwr.; «o » printed; «Typus» printed on pale green paper; "Cloeotus / hamiger / Ohs» handwr. by Bruch, with a red frame; "hamiger Ohaus / Type» handwr. by Ohaus, cut, remainings of « $ᄋ »$. 1 synt. $\sigma^{\pi}$, pinned; «Rep. Argentina / Pr. Santiago d. Estero / 190 / C. Bruch» printed; «ơ" printed; «Typus» printed on pale green paper; «Cloeotus / hamiger / Ohaus» handwr. by Bruch, with a red frame; "hamiger Ohaus / Type» handwr. by Ohaus, cut, remainings of «Cloeotus» and of " $\sigma^{\pi} »$. Both in BR 64. MACN-En 1112 and 1113 respectively.

insperatus [Chaetodus (Pseudohybosorus) amazonicus]. Martínez 1988: 66-69, f. 4: Brasil: Mato Grosso: Tapirapés, holot. $\sigma^{7}$ in MC.

Holot. $\sigma^{7}$, glued to a card, genitalia and abdomen glued to the same card; «Tapirape. Mt. / 30.XII.960 / B. Malkin lg» handwr. on white paper, with a black frame; «Holotipo» handwr. on red paper; «Chaetodus (Chaetodus) / amazonicus $\sigma^{\pi} /$ insperatus ssp. nov. (handwr.) / A. Martínez det. 1987 (printed)» on red paper, with a black frame; "Bs As. / FCO 04/99" printed on yellow paper. MACN-En 1159.

magnificus [Daimothoracodes]. Martínez 1994: 233-235, f. 5-6: Bolivia: Santa Cruz:

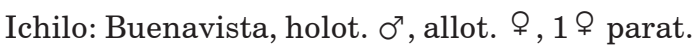
in MC.

Holot. $\sigma^{x}$, glued to a card, genitalia and abdomen glued to the same card; «Bolivia-Sta. / Cruz, Pcia. Ichilo, / Buenavista III-51- / Martínez leg." photographed from typed; "Holotipo" handwr. on red paper; "Daimothoracodes / magnificus $\sigma^{T} /$ sp. nov. (handwr.) / A. Martínez det. 1987 (printed)» on red paper, with a black frame. Allot. $ᄋ$, glued to a card, abdomen on the same card; «Bolivia / Dep. Sta. Cruz / Pcia. Ichilo / Buenavista / Coll. Martínez / Oct. 949» handwr. on white paper; "Allotypus» printed on pale red paper; «Daimothoracodes / magnificus ㅇ / sp. nov. (handwr.) / A. Martínez det. 1987 (printed)» on red paper, with a black frame. MACN-En 1265, 1266 respectively.

monrosi [Acanthocerus]. Martínez \& Pereira 1959: 159-163: Bolivia: Cochabamba: Chapare: S. F. del Chipiriri, holot. $\sigma^{7}$ in MC.

Holot. $\sigma^{7}$, glued to a card, genitalia and abdomen glued to another card; «Bolivia? $\mathrm{D}^{\text {o }}$ Cochab. / Pcia. Chapare - S. F. del / Chipiriri 400 m, I-53 / Martínez-Prosen-col.» photographed from typed; «Holotypus» printed on pale red paper; "Acanthocerus / monrosi o" / sp. n. (handwr.) / A. Martínez det. 1958 (printed)» on red paper, with a black frame. MACN-En 1306.

Note: Currently in Ceratocanthus (Martínez 1967: 12).

punctulatus [Cloeotus]. Ohaus 1911: 555-556: Chaco (Bruch), Misiones (Jörgensen); Brasil: Petrópolis, 16-VIII-1898 (Ohaus).

2 synt. (both?), pinned. One: «Rep. Argentina / Gob. Misiones / 190_/ C. Bruch» printed; «Typus» printed on pale green paper; «Cloeotus / punctulatus Ohs / Ohaus» handwr. by Bruch, with a red frame; "Cloeotus / punctulatus Ohs / Typo (handwr. by Ohaus) / Ohaus determin. 1911 (printed)» on white paper, folded in three. The other one: «Rep. Argentina / Gob. Chaco / 2.1895 / C. Bruch» printed, date handwr.; "Cotypus» printed on pale green paper; "Cloeotus / punctulatus Ohs / Ohaus» handwr. by Bruch, with a red frame; "Cloeotus / punctulat» handwr. by Ohaus. Both in BR 64. MACN-En 1426, 1427 respectively.

Note: Currently in Germarostes Paulian (fide Martínez, letter I, 1993).

similis [Hapalonychoides]. Martínez 1994: 236237: Formosa: Clorinda, holot. 우, 2 ㅇ parat.; Paraguay: Asunción, 19 parat. in MC.

Holot. $\odot$, glued to a card, genitalia and abdomen glued to the same card; «Argentina / For- 
mosa / Clorinda / Coll. Martínez / Dic. 950» handwr. on white paper; «Holotipo» handwr. on red paper; «Hapalonychoides / similis $\$$ / gen. et sp. nov. (handwr.) / A. Martínez det. 1987 (printed)» on red paper, with a black frame. MACN-En 1482.

venezolanus [Chaetodus (Chaetodopsis)]. Martínez 1994: 227-228, f. 2: Venezuela: Aragua: Maracay (El Limón), holot. $\sigma^{7}$ in MC.

Holot. $\sigma^{x}$, glued to a card, genitalia and abdomen glued to the same card; «Venezuela / E ${ }^{\mathrm{o}}$ Aragua / Maracay / El Limón / Coll. Martínez / Jun. 984» handwr. on white paper; «Holotipo» handwr. on red paper; "Chaetodus (illegible) / venezolanus $0^{\top} /$ sp. nov. (handwr.) / A. Martínez det. 1987 (printed)» on red paper, with a black frame. MACN-En 1540.

\section{Lucanidae}

argentinensis [Ceratognathus]. Martínez 1981: 13-19.

Holot. $\sigma^{\pi}$, glued to a card; "Argentina / Neuquen / Lago Curruhe (sic, pro Currhué) Gran/ de, s/ tronco Nothoph. (sic, pro Nothofagus) / J.R. de, H.A. y A. Martínez! / Coll. Martínez / Ene. 967» handwr. on white paper; «Holotypus» printed on pale red paper; "Ceratognathus / argentinensis $\mathrm{O}^{\top} / \mathrm{sp}$. nov. (handwr.) / A. Martínez det. 1978 (printed)» on red paper, with a black frame. MACN-En 1586.

Note: Currently in Hilophyllus Paulsen \& Mondaca.

\section{Trogidae}

hemisphaericus [Trox]. Burmeister 1876: 253254, 264-265: Santa Cruz River in the South of Patagonia, leg. Berg.

9 synt., pinned, without locality label; at the beggining of the series, on the bottom of the drawer in HB a label reads «Omorgus Erichs. Br.», «Trox hemisphaericus Burmeister 1876 Syntypus» handwr. by AOB on pink paper. MACN-En 1117-1125.

Note: Currently in Polynoncus Burmeister.

pampeanus [Trox]. Burmeister 1876: 255-256: All around La Pampa, Tucumán, Catamarca and Rio Cuarto (leg. Burmeister), El Carmen, Patagonia (leg. Berg).

4 synt., pinned. 10 «Carm. / Patag.» printed on green paper. $2^{\mathrm{O}}$ «Cata- / marca» printed on green paper. $3^{\mathrm{o}}$ without locality label. $4^{\mathrm{o}}$ «Tucuman» printed on green paper; «Col. Antigua» printed on pale yellow paper; «68» and
«Paratypus» printed on yellowish orange paper; «pampeanus Nob.» handwr. on white paper and glued on yellow paper with «Museo Argentino de Ciencias Naturales» printed. In all: «Trox pampeanus Burmeister 1876 Syntypus» handwr. by AOB on pink paper. MACN-En 1342, 1345, 1344, 1343 respectively.

Note: Currently in Polynoncus Burmeister.

\section{SPECIMENS NOT FOUND}

beneshi [Sclerostomus, Lucanidae]. Martínez 1953: 43-47, f. 1-2: Jujuy: Trementinal, holot. $0^{7}$ in MC.

Holot. $\sigma^{7}$, glued to a card, genitalia glued to another card; «Argentina / Jujuy / Trementinal / Duret - legit. / Coll. Martínez / Oct. 946» handwr. on white paper; «Holotypus» printed on pale red paper; «Sclerostomus / beneshi $\sigma^{\pi} / \mathrm{sp}$. n. (handwr.) / A. Martínez det. 1953 (printed)» on red paper, with a black frame; «Metadorcinus / beneshi $\sigma^{\pi}$ / (Martínez) (handwr.) / A. Martínez det. 1975 (printed)» on white paper; «Beneshinus / n. gen. (handwr.) / Weinreich det. 1960 (printed)» on white paper.

tucumanus [Sclerostomus, Lucanidae]. Nagel 1932: 119, f. 6 . Argentina.

1 spec., glued to a card; «Argentina / Salta / Dep. Capital / San Lorenzo / Coll. Martínez / Ene. 948" handwr. on white paper; «Allotypus» printed on pale red paper; «Sclerostomus / tucumanus o / Nagel (handwr.) / A. Martínez det. 1953 (printed)» on red paper, with a black frame; «Beneshinus / n. gen. (handwr.) / Weinreich det. 1960 (printed)» on white paper; «Beneshinus / tucumanus 우 / (Nagel) (handwr.) / A. Martínez det. 1975 (printed)» on white paper, with a black frame.

Note: Martínez (1953: 47-48) designated an «allotype».

zischkai [Scorthizus, Lucanidae]. Martínez 1957: 225-232: Bolivia: Cochabamba: prov. Chapare: Yungas del Palmar: Limbo, holot. $\varnothing^{7}$, allot. $ᄋ$ in MC.

Holot. $\sigma^{7}$ and allot. $\odot$, glued to cards. The holot.: «Bolivia / Dep. Cochabamba / Pcia. Chapare / Yungas del Palmar / Zischka - legit. / Coll. Martínez» handwr. on white paper. The allot.: «Bolivia / Yungas del Palmar / 2.000 M. Zischka» printed on white paper, with a grey frame; «10.II.1945» handwr. «Holotypus» and «Allotypus» printed on pale red paper; «Scorthizus / zischkai o" [O ] / sp. n. (handwr.) / A. Martínez det. 1955 (printed)» on red paper, with a black frame. 


\section{NAMES NOT FOUND IN THE BIBLIOGRAPHY \\ AND PROBABLY NOT PUBLISHED}

unicolor [Athyreus flavithorax, Geotrupidae]. Martínez.

Holot. $\sigma^{7}$, glued to a card, genitalia glued to another card; «S. Tomé / Corrientes (printed) / 6.927 (handwr.)»; «Holotypus» printed on pale red paper; "Athyreus / flavithorax / unicolor $\sigma^{\text {" } ~ / ~}$ ssp. n. (handwr.) / A. Martínez det. 1954 (printed) " on red paper, with a black frame. Allot. 옹 glued to a card; «Garruchos / Corrientes / III.1944 Boero» handwr. on white paper, with a black frame; «nov. in B.M. / Athyreus sp. (handwr.) / G.J. Arrow det. (printed)» on white paper; «Allotypus» printed on pale red paper; «Athyreus / flavithorax / unicolor $\odot$ / ssp. n. (handwr.) / A. Martínez det. 1954 (printed) « on red paper, with a black frame. 1 parat. $\sigma^{7}$, pinned, genitalia and abdomen glued to a card; "Catamarca / -1-28 / Bocchio CI.» printed on white paper; «210» printed on white paper; «Paratipo" printed on green paper; «Athyreus / flavithorax / unicolor $\sigma^{x}$ / ssp. n. (handwr.) / A. Martínez det. 1954 (printed) « on green paper, with a black frame. 1 parat. $९$, pinned; "Stgo. Estero / E. Wagner» handwr. by Bruch; «Athyreus / n. sp.?» handwr. by Bruch; «Paratipo» printed on green paper; «Athyreus / flavithorax / unicolor $ᄋ$ / ssp. n. (handwr.) / A. Martínez det. 1954 (printed) « on green paper, with a black frame; in BR 66. 4 parat. $\%$, pinned, 3 with abdomen glued to a card; «S. Tomé / Corrientes (printed) / 11.927 (handwr.)»; «Paratipo» printed on green paper; «Athyreus / flavithorax / unicolor $\odot$ / ssp. n. (handwr.) / A. Martínez det. 1954 (printed) « on green paper, with a black frame. 1 parat. $\sigma^{\pi}$, pinned, genitalia and abdomen glued to a card; «S. Tomé / Corrientes (printed) / 9.926 (handwr.)»; «Paratipo» printed on green paper; «Athyreus / flavithorax / unicolor $\sigma^{\pi} /$ ssp. n. (handwr.) / A. Martínez det. 1954 (printed) « on green paper, with a black frame. MACN-En 1522, 1523, 1515-1521 respectively.

Note: Currently in Neoathyreus (Howden \& Martínez 1978: 53).

\section{SPECIMENS LABELLED AS TYPES BUT NOT INCLUDED IN THE ORIGINAL SERIES}

brasiliensis [Trox, Trogidae]. Vaurie 1962: 134135, f. 14, 67-69: Holot. ơ Brasil: São Paulo: Ypiranga, 01-XII-1937, leg. Lange de Morretes, in MZSP. Paratypes in MZSP, AMHN, USNM, MF and MC. Other specimens from Brasil: Goyaz,
Santa Catharina, Paraná, and Paraguay: Hohenau in MZSP.

1 spec., pinned, «Brasil São Paulo / Rua Maestro Cardim 987» printed on white paper with a black frame, "Paratype / Trox / brasiliensis / P. Vaurie» printed and handwr. on yellow paper. MACN-En 933.

Note: The specimen is not a paratype fide Martínez, letter I, 1993.

chalybeatus [Athyreus, Geotrupidae]. Fairmaire 1892: 242-243: «Plata Orientale».

1 spec., pinned; «Playa Juancho / Bs. Aires / Yepes II.1928» handwr. by Bruch; «Typus» handwr. by Bruch on pale green paper; «Athyreus / chalybeatus / Fairm.» handwr. by Bruch, with «C. Bruch determ.» printed; in BR 66. MACNEn 1039.

Note: The specimen was collected 36 years after the publication date.

quinquedentatus [Bradycinetus, Geotrupidae]. Felsche 1909: 764: Mendoza, O', leg. JensenHaarup.

1 spec., pinned; «Argentina / San Luis / $\mathrm{D}^{\mathrm{o}}$ Capital / Balde / Coll. Martínez / Marz. 946» handwr. on white paper; "Allotypus» printed on pale red paper; «Zefevazia / quinquedentata / (Felsche) (handwr.) / A. Martínez det. 1954 (printed)» on red paper, with a black frame. MACN-En 1436.

Note: The specimen was collected 37 years after the publication date. Currently in Bolborhinum (Zefevazia) (Martínez 1976: 549).

tuberifer [Athyreus, Geotrupidae]. Felsche 1909: 762, pl. X, f. 4.4a: Pernambuco; near $A$. tridens.

1 spec., pinned, abdomen on a card; « Est. Exp. Loreto / (Misiones-Arg.) / Dr. A.A. Oglobin (sic, pro Obloblin)» printed on white paper; «Athyreus / tuberifer $ᄋ$ / Felsche (handwr.) / A. Martínez det. 1952 (printed)» on red paper, with a black frame; «Designación / no válida / (ver descr. orig.)» handwr. on green paper. MACN-En 1508.

Note: Martínez (1953: 233) designated an «allotype», but Howden \& Martínez (1978: 39-41) say the «allotype» was erroneously identified and is not conspecific with the holotype. Moreover, it was designated 44 years after the original publication.

\section{ACKNOWLEDGEMENTS}

The authors would like to thank Martín Ramírez, Pablo Teta and Daniel Rodríguez, for their assistance during this work. The 
digitization of records was supported by a grant from Williams Foundation, Argentina, for the Project of Informatization of the Collections of the MACN. This project was supported, in part, by an NSF-DBI grant (0500767), an NSF Advances in Biological Informatics grant (0743783), and by a PIP-CONICET grant (1122008010-1869) to FCO. FCO thanks CONICET (Comisión Nacional de Investigaciones Científicas y Técnicas) and Instituto de Ciencias Básicas Univ. Nac. de Cuyo for their continuous support to his research.

\section{BIBLIOGRAPHY}

Bruch, C. 1925. Coleópteros nuevos y poco conocidos. Physis, Buenos Aires 8 (29): 199-211.

Burmeister, H. 1876. Die Argentinischen Arten der Gattung Trox Fabr. Stettiner Entom. Zeitg. 37 (79): 241-268.

Fairmaire, L. 1892. Descriptions de quelques Coléoptères argentins. Annls. Soc. Entom. Belgique 36: 242-253.

Felsche, C. 1909. Neue und alte coprophage Scarabaeiden (Col.). Deutsche entom. Zeitschr. 1909: 751-765.

Gutiérrez Alonso, R. 1946. Notas sobre Scarabaeidae chilenos. Livro Homenagem R. Ferreira d'Almeida, S. Paulo, Soc. Brasil. Entom. (2): 11-15.

Howden, H.F. 1973. Bolbothyreus, a new genus for Stenaspidius ruficollis (Coleoptera: Scarabaeidae: Geotrupinae). Canad. Entom. 105 (12): 1567-1571.

Howden, H.F. \& A. Martínez. 1978. A review of the New World genus Athyreus Macleay (Scarabaeidae, Geotrupinae, Athyreini). Contr. Am. Entom. Inst. 15 (4): $1-70$.

International Commission on Zoological Nomenclature. 1999. International Code of Zoological Nomenclature $4^{\text {th }}$ Edit.: XXIX +306 p.

Lawrence, J.F. \& A.F. Newton, Jr. 1995. Families and subfamilies of Coleoptera (with selected genera, notes, references and data on family-group names): 779-1006. En: J. Pakaluk \& S.A. Slipinski (Eds.). Biology, Phylogeny and Classification of Coleoptera. Papers Celebrating the 80th Birthday of Roy A Crowson. Muzeum i Instytut Zoologii PAN. Warsaw, $1092 \mathrm{pp}$.

Luederwaldt, H. 1929. Tres novas especies do gênero Bolboceras (Col. Lamellicornid. Geotrupid.). Bolm. Mus. Nac. R. Jan. 5 (4): 71-72.

Martínez, A. 1947. Insectos nuevos o poco conocidos V (Col. Scarabaeoidea). Rev. Soc. Entom. Arg. 13 (15): 262-280.

- 1951. Insectos nuevos o poco conocidos VIII (Col. Scarabaeidae). Rev. Soc. Entom. Arg. 15: 108-120. 1952. Insectos nuevos o poco conocidos IX. Algunas notas sobre Bolbocerini argentinos y chilenos con descripción de una especie nueva (Col. Scarab. Geotrupinae). Rev. Soc. Entom. Arg. 15: 314-327.

- 1952. Scarabaeidae nuevos o poco conocidos III
(Coleoptera). Publ. Mis. Est. Patol. Region. Arg. 23 (81-82): 53-118.

1953a. Una nueva especie de Athyreus (Col. Scarab. Geotrup.). Mitt. München. Entom. Ges. 43: 226-233.

1953b. Insectos nuevos o poco conocidos X. Algunas notas sobre lucánidos con descripción de una especie nueva y el alotipo de Sclerostomus tucumanus Nagel (Col. Scarabaeoid. Lucanidae). Rev. Soc. Entom. Arg. 16 (2): 42-48.

- 1954. Un nuevo género de Bolboceratini neotropical (Coleoptera, Scarabaeidae). Rev. Bras. Entom. 2: 193-204.

- $\quad$ 1956a. Notas coleopterológicas VII. Anls. Soc. Cient. Arg. 160 (2): 78-97.

- $\quad 1956 b$. Scarabaeoidea neotrópica III. Nuevas especies de Hybosorinae bolivianos (Col.). Neotropica 2 (8): 41-48.

1957. Una nueva especie de Scorthizus (Col. Scar. Lucanidae). Rev. Chil. Entom. 5: 225-232.

1967. Insectos nuevos o poco conocidos XIII. Ceratocanthini nom. nov. para Acanthocerini (Coleoptera, Scarabaeidae, Troginae). Rev. Soc. Entom. Arg. 30: 9-16.

1970. Scarabaeoidea neotropica XVI. Una nueva especie neotropical de Astaenomoechus (Col. Scarab. Troginae Ceratocanthini). Neotropica 15 (48): 112-114.

1976. Contribución al conocimiento de los Bolboceratini Sudamericanos (Coleoptera, Scarabaeidae, Geotrupinae, Bolboceratini). Studia Entom. 19 (14): 531-551.

- 1981. Un nuevo Ceratognathus neotropical (Coleoptera, Lucanidae). Boln. Entom. Venezolana n. s. 2 (2): 13-20.

- 1988. Notas sobre Hybosorinae Sudamericanos I (Col. Scarabaeidae). Anls. Soc. Cient. Arg. 218: 63-69.

- 1994. Notas sobre Hybosorinae (Coleoptera: Scarabaeidae) II. Elytron 8: 223-239.

Martínez, A. \& F.S. Pereira. 1959. Notas sobre Acanthocerinae con descripción de un nuevo género y una nueva especie (Col. Scarabaeidae). Acta Zool. Lilloana 17: 149-164.

Martínez, A.; F.S. Pereira \& M.A. Vulcano. 1961. Glaresini, nueva tribu de Trogidae para la región Neotropical (Coleoptera, Scarabaeoidea). Anls. Soc. Cient. Arg. 171 (1): 67-82.

Nagel, P. 1932. Neues über Hirschkäfer. Entom. Blätter 28: 81-88, 113-121.

Ohaus, F. 1910. Neue Coleoptera lamellicornia aus Argentinien. Deutsche entom. Zeitschr. 1910: 173186.

- 1911. Neue Coleoptera lamellicornia aus Argentinien (Col.). Deutsche entom. Zeitschr. 1911: 553565.

Rodríguez, D. 2007-2010. Aurora. Aplicación para el manejo de bases de datos de colecciones biológicas. Museo Argentino de Ciencias Naturales «Bernardino Rivadavia», CONICET.

Vaurie, P. 1962. A revision of the genus Trox in South America (Coleoptera, Scarabaeidae). Bull. Amer. Mus. Nat. Hist. 124: 107-167. 